\title{
QUANTUM CHAOS IN THE YANG-MILLS-HIGGS SYSTEM AT FINITE TEMPERATURE
}

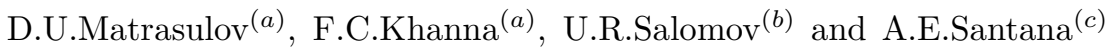 \\ (a) Physics Department University of Alberta \\ Edmonton Alberta, T6G 2J1 Canada \\ and TRIUMF, 4004 Wersbrook Mall, \\ Vancouver, British Columbia, Canada, V6T2A3 \\ (b) Heat Physics Department of the Uzbek Academy of Sciences, \\ 28 Katartal St., 700135 Tashkent, \\ Uzbekistan (c) Instituto de Fisica, \\ Universidade be Brasilia, 70910-900, Brasilia, DF, Brazil
}

(Dated: November 3, 2018)

\begin{abstract}
The quantum chaos in the finite-temperature Yang-Mills-Higgs system is studied. The energy spectrum of a spatially homogeneous $S U(2)$ Yang-Mills-Higgs is calculated within thermofield dynamics. Level statistics of the spectra is studied by plotting nearest-level spacing distribution histograms. It is found that finite temperature effects lead to a strengthening of chaotic effects, i.e. spectrum which has Poissonian distribution at zero temperature has Gaussian distribution at finite-temperature.

PACS numbers: 05.45.-a,11.10.-Wx, 11.15.-q
\end{abstract}

\section{INTRODUCTION}

Quantum chaos is a relatively new area in physics and has been the subject of extensive studies for the last two decades [1]-6]. It has found applications in atomic and molecular physics, nuclear physics and condensed matter physics. In the past few years there is growing interest in quantum chaos in particle physics, too. Being the quantum theory of classically chaotic systems the quantum chaology studies fluctuations in the energy spectra and wave functions of such systems. It is well known, that the energy spectra of systems, whose classical counterparts are chaotic, has the same statistical properties as those for random matrices. Therefore one of the main topics in the quantum chaology is to study the statistical properties of the classically chaotic systems. Recently energy fluctuations and quantum chaos in hadrons and QCD has become a subject of theoretical studies [7]-[12]. In particular, it is found that the quark-gluon system in QCD is governed by quantum chaos in both confined and deconfined phases [8, 9]. The statistical analysis of the measured meson and baryon spectra shows that there is quantum chaos phenomenon in these systems [10]. The study of the charmonium spectral statistics and its dependence on color screening has established quantum chaotic behaviour [7]. It was claimed that such a behaviour could be the reason for $J / \Psi$ suppression [7].

In recent years there has been considerable interest to determine the role of dynamical chaos in field theories [13]-21]. Chaotic properties of Yang-Mills 13, 15], YangMills-Higgs [15]-19] and Abelian Higgs [20] have been treated. The main point in this considerations is the fact that the Hamiltonians of the Yang-Mills and Yang-MillsHiggs systems can be written in the same form as those for the coupled nonlinear oscillators. This allows one to use, for their treatment the same methods as in the case of coupled nonlinear oscillators. Quantum chaos in Yang-Mills-Higgs system was also studied recently [22, 23]. However all works on chaos in field theories and hadrons are restricted to considering zero-temperature cases.

In this paper we study quantum chaos at finitetemperature in the Yang-Mills-Higgs system. Recent advances in heavy ion collision experiments allow one to create hot and dense hadronic and quark-gluon matters. The role of finite-temperature effects in such systems becomes important. Especially in quark-gluon or nuclear matter hadrons behave as complex systems where strong level fluctuations can be observed. Thus the role of finitetemperature effects as well as level fluctuations are crucial in such a systems, that obviously leads to a need for studying quantum chaos at finite-temperature. In particular, finite-temperature effects cause fluctuations in their energy spectra.

We address the problem of heat-bath in quantum chaos through the thermofield dynamics (TFD) formalism, a real time finite temperature field theory 24, 25, 26, 27, 28]. TFD is interesting for our proposal here by its remarkable algebraic structure (this is not the case of the Matsubara 29] or the Schwinger-Keldysh 30] formalisms). Actually the central ideas of TFD involve an algebraic doubling in the degrees of freedom and a Bogoliubov transformation giving rise to the thermal variables. As we will show, this TFD prescription is a useful tool to explore finite-temperature effects in the energy fluctuations of the Yang-Mills-Higgs Hamiltonian, represented in terms of the annihilation and creation operators.

In section II we present the Yang-Mills-Higgs system at zero temperature. In section III the Yang-MillsHiggs system at finite-temperature is studied using the TFD formalism. In section IV we present numerical results for finite-temperature and compare them to zero- 
temperature results, thus bringing out crucial role of finite-temperature in Yang-Mills-Higgs system and quantum chaos. Finally in Section V we present some conclusions and new directions to pursue the important question of quantum chaos.

\section{ZERO TEMPERATURE CASE}

The Lagrangian for Yang-Mills-Higgs system with $S U(2)$ symmetry is given as

$$
L=-\frac{1}{4} F_{\mu \nu}^{a} F_{a}^{\mu \nu}+\frac{1}{2}\left(D_{\mu} \phi\right)^{+}\left(D^{\mu} \phi\right)-V(\phi)
$$

where

$$
\begin{gathered}
F_{\mu \nu}^{a}=\partial_{\mu} A_{\nu}^{a}-\partial_{\nu} A_{\mu}^{a}+g A_{\mu}^{b} A_{\nu}^{c} \\
\left(D_{\mu} \phi\right)=\partial_{\mu} \phi-i g A_{\mu}^{b} T^{b} \phi
\end{gathered}
$$

with $T^{b}=\sigma / 2, b=1,2,3$ generators of the $S U(2)$ algebra and $g$ is a coupling constant. The potential of the scalar (Higgs) field is

$$
V(\phi)=\mu^{2}|\phi|^{2}+\lambda|\phi|^{4},
$$

where $\mu$ and $\lambda$ are constants. Here we give a brief description of the non-thermal case 22]. In $(2+1)$-dimensional Minkowski space and for spatially homogeneous YangMills and Higgs fields which satisfy the conditions

$$
\partial_{i} A_{\mu}^{a}=\partial_{i} \phi=0, \quad i=1,2, ;
$$

and in the gauge $A_{0}^{\alpha}=0$, the Lagrangian can be written as

$$
\begin{gathered}
L=\dot{\vec{\phi}^{2}}+\frac{1}{2}\left(\dot{\vec{A}_{1}^{2}}+\dot{\vec{A}_{1}^{2}}\right)-g\left[\frac{1}{2} \vec{A}_{1}^{2} \vec{A}_{2}^{2}-\frac{1}{2}\left(\overrightarrow{A_{1}} \vec{A}_{2}\right)^{2}+\right. \\
\left.\left(\overrightarrow{A_{1}^{2}}+\vec{A}_{2}^{2}\right) \vec{\phi}^{2}-\left(\overrightarrow{A_{1}} \vec{\phi}\right)^{2}-\left(\overrightarrow{A_{2}} \vec{\phi}\right)^{2}\right]-V(\vec{\phi}),
\end{gathered}
$$

where $\vec{\phi}=\left(\phi^{1}, \phi^{2}, \phi^{3}\right), \vec{A}_{1}=\left(A_{1}^{1}, A_{1}^{2}, A_{1}^{3}\right)$ and $\vec{A}_{2}=$ $\left(A_{2}^{1}, A_{2}^{2}, A_{2}^{3}\right)$

To treat the chaotic dynamics it is convenient to use the canonical formalism and work with the Hamiltonian of the system instead of the Lagrangian. The Hamiltonian of the system can be written as 22.

$$
H=\frac{1}{2}\left(p_{1}^{2}+p_{2}^{2}\right)+g^{2} v^{2}\left(q_{1}^{2}+q_{2}^{2}\right)+\frac{1}{2} g^{2} q_{1}^{2} q_{2}^{2},
$$

where $\vec{\phi}_{0}=(0,0, v) q_{1}=A_{1}^{1}, q_{2}=A_{2}^{2}$ (other components of the Yang-Mills fields are zero) $p_{1}=\dot{q}_{1}$ and $p_{2}=\dot{q}_{2}$, with $\omega^{2}=2 g^{2} v^{2}$ being the mass term of the Yang-Mills field. This is the Hamiltonian of the classical system. Replacing $p_{i}$ and $q_{i}$ by operators and introducing the following creation and destruction operators

$$
\hat{a}_{k}=\sqrt{\frac{\omega}{2}} \hat{q}_{k}+i \sqrt{\frac{1}{2 \omega}} \hat{p}_{k} \quad \hat{a}_{k}^{+}=\sqrt{\frac{\omega}{2}} \hat{q}_{k}-i \sqrt{\frac{1}{2 \omega}} \hat{p}_{k}
$$

we obtain the corresponding quantum Hamiltonian:

$$
H=H_{0}+\frac{1}{2} g^{2} V
$$

where

$$
H_{0}=\omega\left(a_{1} a_{1}^{+}+a_{2} a_{2}^{+}+1\right),
$$

and

$$
V=\frac{1}{4 \omega^{2}}\left(a_{1}+a_{1}^{+}\right)^{2}\left(a_{2}+a_{2}^{+}\right)^{2},
$$

with $\omega^{2}=2 g^{2} v^{2}$ and the operators $\hat{a}_{k}$ and $\hat{a}_{l}^{+}$satisfy the commutation relations $\left[\hat{a}_{k}, \hat{a}_{l}^{+}\right]=\delta_{k l}, \quad k, l=1,2$. The eigenvalues of this Hamitonian are calculated by numerical diagonalization of the truncated matrix of the quantum Yang-Mills-Higgs Hamiltonian in the basis of the harmonic oscillator wave functions [22]. The matrix elements of $H_{0}$ and $V$ are

$$
<n_{1}^{\prime}, n_{2}^{\prime}\left|H_{0}\right| n_{1}, n_{2}>=\omega\left(n_{1}+n_{2}+1\right) \delta_{n_{1}^{\prime} n_{1}} \delta_{n_{2}^{\prime} n_{2}},
$$

and

$$
\begin{gathered}
<n_{1}^{\prime}, n_{2}^{\prime}|V| n_{1}, n_{2}>=\frac{1}{4 \omega^{2}}\left\{\sqrt{n_{1}\left(n_{1}-1\right)} \delta_{n_{1}^{\prime} n_{1}-2}+\right. \\
\left.\sqrt{n_{1}\left(n_{1}-1\right)} \delta_{n_{1}^{\prime} n_{1}+2}+\left(2 n_{1}+1\right) \delta_{n_{1}^{\prime} n_{1}}\right\} \\
\times\left\{\sqrt{n_{2}\left(n_{2}-1\right)} \delta_{n_{2}^{\prime} n_{2}-2}+\right.
\end{gathered}
$$

$$
\left.\sqrt{n_{2}\left(n_{2}-1\right)} \delta_{n_{2}^{\prime} n_{2}+2}+\left(2 n_{2}+1\right) \delta_{n_{2}^{\prime} n_{2}}\right\}
$$

The numerical procedure for diagonalization of this matrix is described by Salasnich [22]. We use the same method in the case of finite-temperature calculations.

\section{FINITE-TEMPERATURE CASE}

To treat quantum chaos in the finite-temperature Yang-Mills-Higgs system, we apply Thermofield Dynamics(TFD). TFD is a real time operator formalism of quantum field theory at finite temperature in which any physical system can be constructed from a temperature dependent vacuum $\mid 0(\beta)>$ which is a pure state. The thermal average of any operator is equal to the expectation value between the pure vacuum state $\mid 0(\beta)>$ defined by applying Bogolyubov transformations to the usual vacuum state . Furthermore, TFD has two main features. The first one is the doubling of the Fock space such that the original Fock space and its double are defined non-tilde and tilde space respectively. All operators are also doubled and the finite-temperature creation and annihilation operators are constructed by Bogoluybov transformation between tilde and non-tilde operators. This is the same 
procedure in writing down the vacuum state at finite temperature. Mathematically, the field operators have the following properties:

$$
\begin{gathered}
\left(A_{i} A_{j} \tilde{)}=\tilde{A}_{i} \tilde{A}_{j},\right. \\
\left(c A_{i}+A_{j} \tilde{)}=c^{*} \tilde{A}_{i}+\tilde{A}_{j},\right. \\
\left(A_{i}^{*} \tilde{)}=\left(\tilde{A}_{i}\right)^{+},\right. \\
\left(\tilde{A}_{i} \tilde{)}=A_{i}\right. \\
{\left[\tilde{A}_{i} A_{j}\right]=0 .}
\end{gathered}
$$

The Yang-Mills-Higgs Hamiltonian in TFD is given as

$$
\hat{H}=H-\tilde{H}
$$

where $H$ is given by eq 4 and $\tilde{H}$ is given as

$$
\tilde{H}=\tilde{H}_{0}+\frac{1}{2} g^{2} \tilde{V}
$$

with

$$
\tilde{H}_{0}=\omega\left(\tilde{a}_{1} \tilde{a}_{1}^{+}+\tilde{a}_{2} \tilde{a}_{2}^{+}+1\right)
$$

and

$$
\tilde{V}=\frac{1}{4 \omega^{2}}\left(\tilde{a}_{1}+\tilde{a}_{1}^{+}\right)^{2}\left(\tilde{a}_{2}+\tilde{a}_{2}^{+}\right)^{2}
$$

It has been established [31] that in an algebraic approach the doubled set of operators may be considered as a set of operators that relate to the physical observables, $O$, and a second set that are generators of symmetries, $\hat{O}$. The hat operators are responsible, in particular, for time development and are needed for the purpose of scattering, decay and any transitions between states. The physical observables lead to the quantities that are measured in experiment. Both for physical observables and generators of symmetry after Bogolyubov transformations leading to finite-temperature creation and annihilation operators and to a pure vacuum state only the non-tilde operators are required for getting the appropriate matrix elements. However, it is clear that for an analysis of any system at finite temperature both set of operators, $O$ and $\hat{O}$, are needed since it is important to generate the appropriate symmetry of the system in any time development while considering the matrix element we have decided to investigate the chaotic behaviour for the Hamiltonian that relates to physical observables, $H$ and the Hamiltonian that generates the symmetry of the system, $\hat{H}$. Results of the finite-temperature quantum chaos in Yang-Mills-Higgs system will be displayed for the physical observables, and for the generators of symmetry, $\hat{H}$. Only then we will draw conclusions about the approach of quantum chaotic behaviour in the finite-temperature quantum field theory case of Yang-Mills-Higgs theory.

First we need to rewrite the non-tilde part of the Hamiltonian in the temperature-dependent form using the Bogolyubov transformations which are given by

$$
\begin{aligned}
& a_{k}=a_{k}(\beta) \cosh \theta+\tilde{a}_{k}^{+}(\beta) \sinh \theta \\
& a_{k}^{+}=a_{k}^{+}(\beta) \cosh \theta+\tilde{a}_{k}(\beta) \sinh \theta \\
& \tilde{a}_{k}=a_{k}^{+}(\beta) \sinh \theta+\tilde{a}_{k}(\beta) \cosh \theta \\
& \tilde{a}_{k}^{+}=a_{k}(\beta) \sinh \theta+\tilde{a}_{k}^{+}(\beta) \cosh \theta
\end{aligned}
$$

where

$$
\beta=\frac{\omega}{k_{B} T}
$$

where tilde and non-tilde creation and annihilation operators satisfy the following commutation relations:

$$
\left[a_{k}(\beta), a_{l}^{+}(\beta)\right]=\delta_{k l} \quad\left[\tilde{a}_{k}(\beta), \tilde{a}_{l}^{+}(\beta)\right]=\delta_{k l}
$$

$l, k=1,2$, and $\sinh ^{2} \theta=\left(e^{\beta}-1\right)^{-1}$. All other commutation relations are zero.

Then the temperature-dependent forms of $H_{0}$ and $\tilde{H}_{0}$ are

$$
\begin{gathered}
H_{0}=\omega\left\{\left(F_{1}+F_{2}\right) \cosh ^{2} \theta+\right. \\
\left.\left(L_{1}+L_{2}\right) \sinh ^{2} \theta+\left(S_{1}+S_{2}\right) \cosh \theta \sinh \theta+1\right\} \\
\tilde{H}_{0}=\omega\left\{\left(F_{1}+F_{2}\right) \sinh ^{2} \theta+\right. \\
\left.\left(L_{1}+L_{2}\right) \cosh ^{2} \theta+\left(S_{1}+S_{2}\right) \cosh \theta \sinh \theta+1\right\}
\end{gathered}
$$

where

$$
\begin{gathered}
F_{k}=a_{k}^{+}(\beta) a_{k}(\beta), \\
L_{k}=\tilde{a}_{k}(\beta) \tilde{a}_{k}^{+}(\beta), \\
S_{k}=a_{k}^{+}(\beta) \tilde{a}_{k}^{+}(\beta)+\tilde{a}_{k}^{+}(\beta) a_{k}(\beta),
\end{gathered}
$$

For $V$ and $\tilde{V}$ we have

$$
\begin{gathered}
V=\frac{1}{4 \omega^{2}}\left\{A_{1} \cosh { }^{2} \theta+B_{1} \cosh \theta \sinh \theta+\right. \\
\left.C_{1} \sinh ^{2} \theta\right\}\left\{A_{2} \cosh ^{2} \theta+B_{2} \cosh \theta \sinh \theta+C_{2} \sinh ^{2} \theta\right\} \\
\tilde{V}=\frac{1}{4 \omega^{2}}\left\{A_{1} \sinh ^{2} \theta+B_{1} \cosh \theta \sinh \theta+C_{1} \cosh ^{2} \theta\right\} \times
\end{gathered}
$$




$$
\times\left\{A_{2} \sinh ^{2} \theta+B_{2} \cosh \theta \sinh \theta+C_{2} \cosh ^{2} \theta\right\},
$$

where

$$
\begin{gathered}
A_{k}=\left(a_{k}(\beta)+a_{k}^{+}(\beta)\right)^{2}, \\
B_{k}=\left(a_{k}(\beta)+a_{k}^{+}(\beta)\right)\left(\tilde{a}_{k}^{+}(\beta)+\tilde{a}_{k}(\beta)\right)+ \\
\left(\tilde{a}_{k}^{+}(\beta)+\tilde{a}_{k}(\beta)\right)\left(a_{k}(\beta)+a_{k}^{+}(\beta)\right),
\end{gathered}
$$

and

$$
C_{k}=\left(\tilde{a}_{k}^{+}(\beta)+\tilde{a}_{k}(\beta)\right)^{2} .
$$

In the first approach the energy eigenvalues of the thermal Yang-Mills-Higgs system can be calculated by diagonolization of the following matrix:

$$
R=<n_{1}^{\prime} n_{2}^{\prime}, \tilde{n}_{1}^{\prime} \tilde{n}_{2}^{\prime}\left|H_{0}+\frac{1}{2} g^{2} V\right| n_{1} n_{2}, \tilde{n}_{1} \tilde{n}_{2}>.
$$

The elements of the matrix can be calculated explicitly:

$$
\begin{gathered}
<n_{1}^{\prime} n_{2}^{\prime}, \tilde{n}_{1}^{\prime} \tilde{n}_{2}^{\prime}\left|H_{0}\right| n_{1} n_{2}, \tilde{n}_{1} \tilde{n}_{2}>= \\
=\omega\left\{\left(n_{1}+n_{2}+1\right)\left(1+2 \sinh ^{2} \theta\right) \delta_{n_{1}^{\prime} n_{1}} \delta_{n_{2}^{\prime} n_{2}}+\right. \\
\left\{n_{1} \delta_{n_{1}^{\prime} n_{1}-1} \delta_{n_{2}^{\prime} n_{2}}+n_{2} \delta_{n_{2}^{\prime} n_{2}-1} \delta_{n_{1}^{\prime} n_{1}}+\left(n_{1}+1\right) \delta_{n_{1}^{\prime} n_{1}+1} \delta_{n_{2}^{\prime} n_{2}}+\right. \\
\left.\left.\left.\left(n_{2}+1\right) \delta_{n_{2}^{\prime} n_{2}+1} \delta_{n_{1}^{\prime} n_{1}}\right)\right\} \cosh \theta \sinh \theta\right\}
\end{gathered}
$$

and for $V$

$$
\begin{gathered}
<n_{1}^{\prime} n_{2}^{\prime}, \tilde{n}_{1}^{\prime} \tilde{n}_{2}^{\prime}|V| n_{1} n_{2}, \tilde{n}_{1} \tilde{n}_{2}>= \\
\frac{1}{4 \omega^{2}}<n_{1}^{\prime} n_{2}^{\prime}, \tilde{n}_{1}^{\prime} \tilde{n}_{2}^{\prime} \mid\left\{A_{1} A_{2} \cosh ^{4} \theta+C_{1} C_{2} \sinh ^{4} \theta+\right. \\
\left(A_{1} C_{2}+B_{1} B_{2}+C_{1} A_{2}\right) \cosh ^{2} \theta \sinh ^{2} \theta+ \\
\left(A_{1} B_{2}+B_{1} A_{2}\right) \cosh ^{3} \theta \sinh \theta+ \\
\left.\left(B_{1} C_{2}+C_{1} B_{2}\right) \cosh \theta \sinh ^{3} \theta\right\} \mid n_{1} n_{2}, \tilde{n}_{1}, \tilde{n}_{2}>
\end{gathered}
$$

The matrix elements of $A_{k}, B_{k}$ and $C_{k}$ are given as

$$
\begin{gathered}
<n_{1}^{\prime} n_{2}^{\prime}, \tilde{n}_{1}^{\prime} \tilde{n}_{2}^{\prime}\left|A_{k}\right| n_{1} n_{2}, \tilde{n}_{1} \tilde{n}_{2}>= \\
=\sqrt{n_{k}\left(n_{k}-1\right)} \delta_{n_{k}^{\prime} n_{k}-2}+\left(2 n_{k}+1\right) \delta_{n_{k}^{\prime} n_{k}}+ \\
+\sqrt{\left(n_{k}+1\right)\left(n_{k}+2\right)} \delta_{n_{k}^{\prime} n_{k}+2},
\end{gathered}
$$

$$
\begin{gathered}
<n_{1}^{\prime} n_{2}^{\prime}, \tilde{n}_{1}^{\prime} \tilde{n}_{2}^{\prime}\left|B_{k}\right| n_{1} n_{2}, \tilde{n}_{1} \tilde{n}_{2}> \\
=2 n_{k} \delta_{n_{k}^{\prime} n_{k}-1}+\left(2 n_{k}+1\right) \delta_{n_{k}^{\prime} n_{k}+1},
\end{gathered}
$$

and

$$
\begin{gathered}
<n_{1}^{\prime} n_{2}^{\prime}, \tilde{n}_{1}^{\prime} \tilde{n}_{2}^{\prime}\left|C_{k}\right| n_{1} n_{2}, \tilde{n}_{1} \tilde{n}_{2}>= \\
=\sqrt{n_{k}\left(n_{k}-1\right)} \delta_{n_{k}^{\prime} n_{k}-2}+\left(2 n_{k}+1\right) \delta_{n_{k}^{\prime} n_{k}}+ \\
\sqrt{\left(n_{k}+1\right)\left(n_{k}+2\right)} \delta_{n_{k}^{\prime} n_{k}+2} .
\end{gathered}
$$

The calculation of the matrix element of $\hat{H}$, the generator of symmetry, gives us the following matrix:

$$
\begin{gathered}
Z=<n_{1}^{\prime} n_{2}^{\prime}, \tilde{n}_{1}^{\prime} \tilde{n}_{2}^{\prime}|H-\tilde{H}| n_{1} n_{2}, \tilde{n}_{1} \tilde{n}_{2}>= \\
=\omega\left\{-\left(2 \cosh ^{2} \theta\right) \delta_{n_{1}^{\prime} n_{1}} \delta_{n_{2}^{\prime} n_{2}}+\right. \\
\left.+\left(n_{1}+n_{2}\right) \delta_{n_{1}^{\prime} n_{1}} \delta_{n_{2}^{\prime} n_{2}}\right\}+ \\
+\frac{g^{2}}{2 \omega^{2}} \cosh ^{4} \theta\left\{\sqrt{n_{1}\left(n_{1}-1\right)} \delta_{n_{1}^{\prime} n_{1}-2}+\left(2 n_{1}+1\right) \delta_{n_{1}^{\prime} n_{1}}+\right. \\
\left.+\sqrt{\left(n_{1}+1\right)\left(n_{1}+2\right)} \delta_{n_{1}^{\prime} n_{1}+2}\right\}
\end{gathered}
$$

Diagonalizing the matrices $R$ and $Z$ numerically we obtain the energy eigenvalues of the Yang-Mills-Higgs system for the Hamiltonians $H$ and $\hat{H}$ at finite-temperature. As it was mentioned [19] the numerical energy levels depends on the dimension of the truncated matrix. We compute the numerical levels in double precision. The matrix dimension is $1156 \times 1156$, i.e. we calculate the first 1156 eigenvalues.. Then the statistical properties of the spectra are found. We use standard unfolding procedure in order to remove the secular variation of the level density as a function of the energy $E$, for each value of the coupling constant the corresponding spectrum is mapped, by a numerical procedure described in [33.

One of the main characteristics of the statistical properties of the spectra is the level spacing distribution [2, 3] function. In this work we calculate the nearest-neighbor level-spacing distribution [2, 3, 4, 7]. The nearest neighbor level spacings are defined as $S_{i}=\tilde{E}_{i+1}-\tilde{E}_{i}$, where $\tilde{E}_{i}$ are the energies of the unfolded levels, which are obtained by the following way: The spectrum $\left\{E_{i}\right\}$ is separated into smoothed average part and fluctuating parts. Then the number of the levels below $E$ is counted and the following staircase function is defined:

$$
N(E)=N_{a v}(E)+N_{\text {fluct }}(E) .
$$


The unfolded spectrum is finally obtained with the mapping

$$
\tilde{E}_{i}=N_{a v}\left(E_{i}\right) .
$$

Then the nearest level spacing distribution function $P(S)$ is defined as the probability of $S$ lying within the infinitesimal interval $[S, S+d S]$.

For the quantum systems which are chaotic in the classical limit this distribution function is the same as that of the random matrices 2, 4]. For systems which are regular in the classical limit its behaviour is close to a Poissonian distribution function. This distribution is usually taken to be a Gaussian with a parameter $d$ :

$$
P(H) \sim \exp \left(-\operatorname{Tr}\left\{H H^{+}\right\} / 2 d^{2}\right),
$$

and the random matrix ensemble corresponding to this distribution is called the Gaussian ensemble. For Hamiltonians invariant under rotational and time-reversal transformations the corresponding ensemble of matrices is called the Gaussian orthogonal ensemble (GOE). It was established [2, 3, 4] that GOE describes the statistical fluctuation properties of a quantum system whose classical analog is completely chaotic. The nearest neighbor level spacing distribution for GOE is described by the Wigner distribution:

$$
P(S)=\frac{1}{2} \pi S \exp \left(-\frac{1}{4} \pi S^{2}\right) .
$$

The usual way to study the level statistics is to compare the calculated nearest-neighbor level-spacing distribution histogram with the Wigner distribution.

For systems whose classical motion is neither regular nor fully chaotic (mixed dynamics) the level spacing distribution will be intermediate between the Poisson and GOE limits. Several empirical functional forms for the distribution have been suggested for this case [4]. If the Hamiltonian is not time-reversal invariant, irrespective of its behavior under rotations, the Hamiltonian matrices are complex Hermitian and the corresponding ensemble is called Gaussian unitary ensemble (GUE). If the Hamiltonian of the system is time-reversal invariant but not invariant under rotations, then the corresponding ensemble is called the Gaussian symplectic ensemble. In the next section we will study the numerical results for the level spacing and then analyze them to classify them in one of the these categories. It is to be emphasized that our Yang-Mills-Higgs Hamiltonian is time-reversal and rotational invariant.

\section{NUMERICAL RESULTS}

On diagonalizing the vacuum expectation value of the Hamiltonian $H$, physical observables, and $\hat{H}$, the generator of symmetry, given in Eq. [7] and Eq. 9] respectively the spectra are analyzed by considering the level-spacing distribution. Then the criterion mentioned in the last section allows us to classify the system as chaotic or nonchaotic. In Figs. 1 and 2 we plot the level spacing distributions for different values of parameters $\omega$ and $\theta$ for the energy spectrum calculated by diagonalizing of the matrix $R$. In Fig. 1 this distribution is given for the value $\omega=0.92$ for which the non-thermal case level spacing distribution is chaotic 22]. As is seen from this figure for $\theta=0$ it is the same as the results of non-thermal calculations 22. By increasing the temperature it becomes closer to a Gaussian distribution that means strengthening of chaos in the thermal case. In Fig. 2 the level spacing distribution for $\omega=0.01$ is plotted. For this value of $\omega$ the systems is regular at $\theta=0$. However the increase of temperature leads to a chaotization of the system and $P(S)$ becomes closer to the Gaussian distribution. Figs. 3 and 4 present level spacing distributions (for the same values of parameters as in Figs 1 and 2) for the spectrum calculated by diagonalizing of the matrix $Z$. By comparing Figs. 1, 2, 3 and 4, it is clear that at zero temperature level spacing distributions are the same for both methods. By increasing the temperature, the difference between spacing distributions for different methods becomes considerable. It is clear from Figs. 1 and 2, that the level spacing distribution for $\omega=0.92$, $\theta=0.01$ is closer to the Gaussian distribution compared to ones for $\omega=0.01, \theta=0.01$. The same behavior can be seen in histograms for $\omega=0.92, \theta=0.2$ and $\omega=0.01$, $\theta=0.2$. The reason for such behavior can be understood from Fig. 5 where temperature $T$ is plotted as a function of $\theta$ for various values of $\omega$. It is clear that higher values of $\omega$ correspond to higher temperatures, while for smaller values of $\omega$ temperature is also small.

However, for $\omega=0.92$ the difference between the results for $H$ and $\hat{H}$ is small while for $\omega=0.01$ there is considerable difference in the results even for $\theta=0.01$ (see Fig. 2 and 4). This indicates that the results from $H$, physical observables and $\hat{H}$, generators of symmetry, are quite similar at high temperature while at low temperature the results are quite different. We know that at zero temperature $H$ and $\hat{H}$ perform different functions. But the results indicate that at high temperature their chaotic behavior is similar.

Thus in both cases increasing the temperature leads to a smooth transition from a Poissonian to a Gaussian form in the level spacing distribution. Furthermore, at higher temperatures both $H$ and $\hat{H}$ lead to quite similar results.

\section{CONCLUSION}

Summarizing we have treated quantum chaos in gauge fields at finite temperature using a toy model, $S U(2)$ Yang-Mills-Higgs system. To account for the finite temperature effects we used the thermofield dynamics technique. The need for simultaneous exploration of the level fluctuations and the finite-temperature effects is dictated by recent advances in relativistic heavy ion collision 
experiments, that allow the creation of hot and dense quark-gluon and hadronic matters 32].

The lattice QCD calculations of hadronic matter and quark-gluon matter indicate that both systems exhibit strong chaotic dynamics [8, 11]. The present calculations in a toy model appear to support such conclusions. Furthermore the study of the Yang-Mills-Higgs system at finite temperature establishes clearly that an increase of temperature of the system strengthens level fluctuations in the spectra. Then a transition from the Poissonian to a Gaussian level-spacing distribution does occur. It is to be anticipated that a study of the quark-gluon system in the relativistic heavy ion collisions at RHIC and later on at LHC would show the phenomenon of quantum chaos in a quantum field theoretic system. It is to be emphasized that a proper study in $(3+1)$ dimensions with full details of the Yang-Mills non-abelian field along with the Higgs scalar field is needed to make necessary conclusions for a quark-gluon plasma. However the present study provides an indication of the possible outcome in more realistic studies.

\section{Acknowledgments}

The work of DUM is supported by NATO Science Fellowship of Natural Science and Engineering Research Council of Canada (NSERCC). The work of FCK is supported by NSERCC. The work of URS is supported by a Grant of the Uzbek Academy of Sciences (contract No 38-02). The work of AES is supported by CNPQ (Brazil).
[1] T. A. Brody et al, Rev. Mod. Phys. 53358 (1981).

[2] B. Eckhardt, Phys. Rep. 163207 (1988).

[3] M. C. Gutzwiller, Chaos in classical and quantum systems. New York, Springer Verlag 1990.

[4] T. Guhr, A. Mueller-Groeling, and H. A. Weidenmueller, Phys. Rep. 299189 (1998).

[5] L. A. Bunimovich, Commun. Math. Phys., 65259 (1979).

[6] G. M. Zaslavsky, Stochastic Behaviour of Dynamical Systems. New York, Harwood. 1985.

[7] Jian-zhong Gu et al, Phys. Rev. C, 60035211 (1999).

[8] M. A. Halasz and J. J. M. Verbaarschot, Phys. Rev. Lett., 743920 (1995).

[9] E. Bittner, H. Markum and R. Pullrich Quantum chaos in physical systems: from superconductors to quarks. hep-lat/0110222

[10] V. Pascalusta, hep-ph/0201040

[11] B. A. Berg, et.al hep-lat/0007008

[12] D. Bukta, G. Karl, B. G. Nickel chao-dyn/9910026

[13] G. K. Savvidy, Phys. Lett. 130B 303 (1983).

[14] G. K. Savvidy, Nucl. Phys. B246 302 (1984).

[15] G. K. Savvidy, Phys. Lett. 159B 325 (1985).

[16] T. Kawabe and S. Ohta, Phys. Lett. 334B 127 (1994).

[17] T. Kawabe and S. Ohta, Phys. Rev. D, 441274 (1991).

[18] L. Salasnich, Phys. Rev. D, 526189 (1995).

[19] L. Salasnich, Mod. Phys. Lett. A 103119 (1995).

[20] T. Kawabe, Phys. Lett. 343B 254 (1995).

[21] S. G. Matinyan and B. Muller, Phys. Rev. Lett., 782515
(1997).

[22] L. Salasnich, Mod. Phys. Lett. A 121473 (1997).

[23] L. Salasnich J. Math. Phys. 404429 (1999)

[24] H. Umezawa, H. Matsumoto and M. Tachiki Thermofield Dynamics.(North-Holland. Amsterdam, 1982)

[25] Y. Takahashi and H. Umezawa, Collective Phenomena 2 55 (1975) (Reprinted in Int. J. Mod. Phys. A 101755 (1996)).

[26] A. Das, Finite Temperature Field Theory (World Scientific, New York, 1997).

[27] Y. X. Gui, F. C. Khanna and Z. B. Su (Eds.) Thermo Field Theories and Their Applications.(World Scientific, Singapore, 1996)

[28] A. Rakhimov and F. C. Khanna, Phys. Rev. C, 64064907 (2001).

[29] T. Matsubara, Prog. Theor. Phys. 14351 (1955).

[30] J. Schwinger, J. Math. Phys. 2407 (1961); L. V. Keldysh, Sov. Phys. JETP 201018 (1964).

[31] A. E. Santana and F. C. Khanna, Phys. Lett. A, 20368 (1995).

[32] Quark Matter 2001, Proceedings of the 15th International Conference on Ultra-Relativistic Heavy Ion Collisions, edited by T. J. Hallman, D. E. Kharzeev, J. T. Mitchell and T .Ullrich (North-Holland, Amasterdam, 2002); Nucl. Phys. A698 1 (2002)

[33] V. R. Manfredi, Lett. Nuouo Cimento 40135 (1984). 